\title{
Simultaneous adsorption of cationic and anionic dyes using a novel multifunctional mesoporous silica
}

\author{
Amir Vahid a,", Majid Abdouss ${ }^{\text {b, }}$, Shahnaz Nayeri ${ }^{\text {b }}$, Aliakbar Miran Beigi a \\ ${ }^{a}$ Research institute of petroleum industry, Tehran, Iran \\ ${ }^{b}$ Faculty of chemistry, Amirkabir University of Technology, Tehran, Iran
}

\section{A R T I C L E I N F O :}

Received 17 Nov 2020

Revised form 22 Jan 2021

Accepted 15 Feb 2021

Available online 29 Mar 2021

Keywords:

Adsorption,

Multi-Functional,

Dye,

Mesoporous silica,

Synthesis.

\begin{abstract}
A B S T R A C T
In the present work a multifunctional nanoadsorbent was synthesized via a well-designed stepwise route, led to the grafting of an amine group on the interior and acidic sites on the exterior of bimodal mesoporous silica nanoparticles (UVM-7). First, amine and thiol groups were grafted on the interior and exterior pores of silica through co-condensation and post synthesis treatment, respectively. Then, the oxidation of thiol on UVM-7 caused to create sulfonic acid and the subsequent template extraction was carried out to obtain the $\mathrm{NH}_{2} / \mathrm{UVM}-7 / \mathrm{SO}_{3} \mathrm{H}$. The results of XRD, the nitrogen sorption, SEM, TEM, FT-IR and elemental analysis revealed the presence of both types of functional groups on UVM-7. Then, simultaneous adsorption of anionic and cationic dyes (Methylene Blue [MB] and Direct Red 23 [Dr]) using $\mathrm{NH}_{2} / \mathrm{UVM}-7 / \mathrm{SO}_{3} \mathrm{H}$ was investigated. UVVis spectrophotometry was utilized for the determination of dyes in single and binary solutions. Langmuir and Freundlich models were used for the fitting of obtained experimental adsorption data and the constants of both isotherms were calculated for MB and Dr. Morover, the calculation of thermodynamic parameters revealed that the adsorption of $\mathrm{MB}$ and $\mathrm{Dr}$ on $\mathrm{NH}_{2} / \mathrm{UVM}-7 / \mathrm{SO}_{3} \mathrm{H}$ was endothermic and spontaneous. Furthermore, the simultaneous adsorption of both dyes regardless of their different electrostatic charge is the main characteristics of $\mathrm{NH}_{2} / \mathrm{UVM}-7 / \mathrm{SO}_{3} \mathrm{H}$ which was specially used for treatment of industrial wastewater.
\end{abstract}

\section{Introduction}

Dyes as a important raw material were used in different industries including cloth, plastics, tanning, cosmetics and food [1]. Additionally, the dyes are one of the most problematic groups as a results of their environmental impact. Furthermore, the industrial effluents containing dyes may be have a carcinogenic effect in humans when, discharge to waters without any proper treatment.

\footnotetext{
*Corresponding Author: Amir Vahid

Email: avahid753@gmail.com \& vahida@ripi.ir https://doi.org/10.24200/amecj.v4.i01.127
}

Methylene Blue (MB) and Direct red (Dr) as the cationic and anionic dyes have harmful effects on human life and environment. Acute exposure to MB causes many health effect such as increased heart rate and cyanosis jaundice quadriplegia in mammals [2,3]. Up to now, the dye adsorption studies have focused on solutions containing single dye and mixture of dyes [4-6]. The most industrial effluents include a mixture of several dyes, so it is necessary to study the simultaneous adsorption of two or more dyes from aqueous solutions [7,8]. However, the dyes are resistance to biodegradation due to their aromatic structure [9]. Many industrial 
technologies, including chemical oxidation [10], adsorption [11], the coagulation/flocculation [12], the membrane separation and ion exchange [13] was used for the adsorption of dyes from industrial effluents. Among them, adsorption has been known as one of the practical physical processes for the treatment and cleaning of wastewaters, because it is cost effective and very effective. The main problem of this technology is the synthesis of low cost adsorbent possesses with high adsorption capacity [14]. In multi-dye adsorption, the interference of one dye on the other dyes is very important [15]. The liquid chromatography [16], the capillary electrophoresis [17], the spectrometry [18] and the electrochemistry methods [19] are used for the analysis of dyes. Due to advantages of UV-Vis spectrophotometry such as accuracy of results, sensitivity, low cost, easy operation and reproducibility, it was used for analysis of colored samples [20]. The main drawback of this technique is the overlap and interference of broad absorption peaks. So, the simultaneous analysis of mixture dyes could be carried out using derivative spectrophotometry [21]. It was determined based on the derivative values of interest compound while other components have zero value [22].
Also, many applications were used by UVM7 in different sciences [23-26]. In this work, the synthesis of a multifunctional mesoporous silica contains both acidic and basic functional groups were reported. At the second step, the adsorption of MB / Dr from aqueous solution was carried out simultaneously and the adsorption phenomenon was studied.

\section{Experimental}

\subsection{Reagents and materials}

All reagents with ultra-trace analytical grade such as; lead nitrate salt, acids and base solutions were purchased from Merck (Darmstadt, Germany). The structure of MB and Dr are shown in Figure 1. Ultrapure water has been obtained from Millipore continental water system (Bedford, USA). Tetraethyl orthosilicate (TEOS, $\left(\mathrm{C}_{2} \mathrm{H}_{5} \mathrm{O}\right)_{4} \mathrm{Si}$, CAS N: 78-10-4, Sigma), triethanolamine $\left(\mathrm{TEAH}_{3}, \mathrm{C}_{6} \mathrm{H}_{15} \mathrm{NO}_{3}, \mathrm{CAS} \mathrm{N}:\right.$ 102-71-6, Sigma), cetyltrimethylammonium bromide (CTAB, $\mathrm{C}_{19} \mathrm{H}_{42} \mathrm{BrN}$, CAS N: 57-09-0, Merck) and other reagents with analytical grade were prepared from Merck or Sigma Aldrich, Darmstadt, Germany. The $\mathrm{pH}$ adjustments were made using appropriate buffer solutions (Merck, Germany).

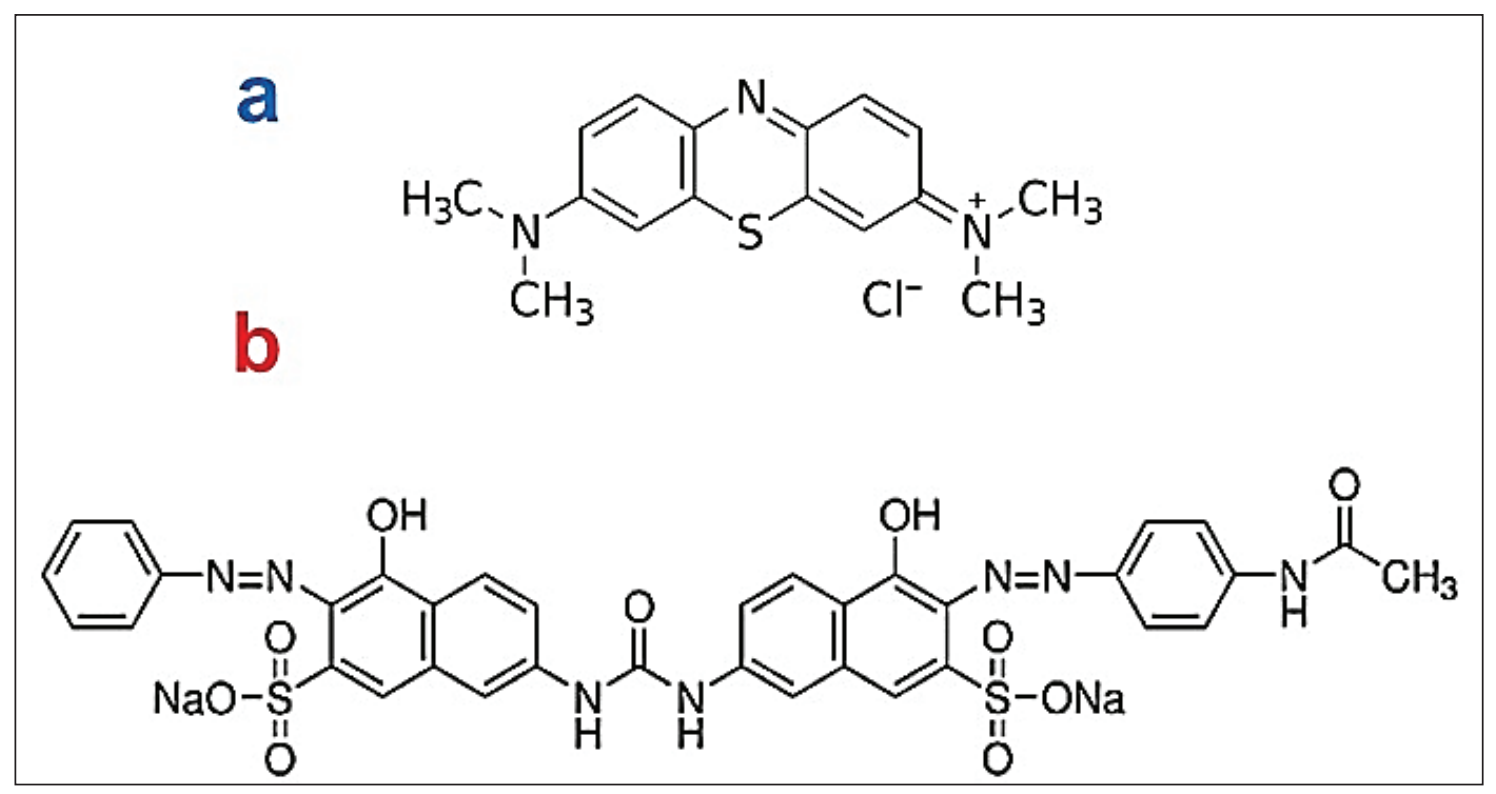

Fig.1. The structure of $\mathrm{MB}(\mathrm{a})$ and $\operatorname{Dr}(\mathrm{b})$ 


\subsection{Synthesis of UVM-7}

UVM-7 was synthesized via well-known atrane route in which triethanol amine (TEAH3), has effect on the rate of hydrolysis and condensation of teraethyl orthosilicate (TEOS). In a typical synthesis, mixture of TEOS and TEAH3 heated up to $120^{\circ} \mathrm{C}$ and then CTAB (cetyltrimethylammonium bromide) was added when the temperature of the solution reached to $70^{\circ} \mathrm{C}$. After addition of water a suspension was formed and aged for 4 hours at ambient temperature. The final molar composition of the synthesis mixture was 1.0 TEOS : 3.5 TEAH3 : 0.25 CTAB : $90 \mathrm{H}_{2} \mathrm{O}$. The product was filtered, washed with water and acetone and dried in an oven at $80^{\circ} \mathrm{C}$ overnight and calcined at $550^{\circ} \mathrm{C}$ for 6 hours.

\subsection{Synthesis of $\mathrm{NH}_{2}-/ \mathrm{UVM}-7 / \mathrm{SO}_{3} \mathrm{H}$}

First, $1.0 \mathrm{~g}$ of aminopropyle triethoxysilane (APTES) was added to the aqueous solution of CTAB and after 5 minutes, mixture of TEOS and TEAOH, was added to the micellar solution. The reactants molar ratio was 1.0 TEOS: 3.5 TEAH3 : $0.25 \mathrm{CTAB}: 90 \mathrm{H}_{2} \mathrm{O}: 0.20$ APTES. After aging, the white suspension was filtered and washed thoroughly with water and acetone. The white precipitate dried in oven over night at $80^{\circ} \mathrm{C}$. At the second step, $1.0 \mathrm{~g}$ of the as-synthesized $\mathrm{NH}_{2} / \mathrm{UVM}$ 7 and $1.0 \mathrm{~g}$ of triethoxysilane propanethiol (TPTES) was refluxed in $50 \mathrm{~mL}$ of toluene for 12 hours and then, the mixture filtered and dried in oven again. Then, thiol groups grafted on the external surface of the sample was oxidized in $15 \% \mathrm{H}_{2} \mathrm{O}_{2}$ and 1 molar solution of $\mathrm{H}_{2} \mathrm{SO}_{4}$. Then, CTAB of the assynthesized $\mathrm{NH}_{2} / \mathrm{UVM}-7 / \mathrm{SO}_{3} \mathrm{H}$ was removed by reflux in 1 molar ethanolic solution of HCL for 24 hours to get access to the internal pores and amine groups. The final product was dried in oven at 80 ${ }^{\circ} \mathrm{C}$ in vacuum oven overnight.

\subsection{Batch adsorption procedure}

In each test, $0.1 \mathrm{~g}$ of adsorbent was added to the 10 $\mathrm{mL}$ of a solution containing a known concentration of each dyes while agitating at $250 \mathrm{rpm}$ at $25^{\circ} \mathrm{C}$. After 120 minutes the concentration of residual dyes in supernatant was determined by UV-Vis spectrophotometer at 502 and $664 \mathrm{~nm}$ for Dr and $\mathrm{MB}$, respectively. In binary solution, the best wavelength for each dye was find out utilizing firstorder derivative spectrometry.

The adsorption percentage ( $\mathrm{R} \%)$ and adsorption capacity of each dye $q_{\mathrm{e}}$, (mg dye/g adsorbent) was calculated according to the following equations (Eq.1 and Eq.2):

$$
\begin{gathered}
q_{e}=\frac{\left(C_{0}-C_{e}\right) V}{M} \\
\text { (Eq. 1) } \\
R \%=\frac{\left(C_{0}-C_{e}\right)}{C_{0}} \times 100
\end{gathered}
$$

(Eq. 2)

Where $\mathrm{C}_{0}$ and $\mathrm{C}_{\mathrm{e}}\left(\mathrm{mg} \mathrm{L}^{-1}\right)$ are the initial and equilibrium concentration of dye, respectively. $V$ (L) is the volume of the solution and $M(\mathrm{~g})$ is the mass of adsorbent used.

\subsection{Characterization}

$\mathrm{X}$-ray diffraction (XRD) patterns were recorded using a Philips 1840 diffractometer using nickelfiltered $\mathrm{Cu} \mathrm{K} \alpha\left(1.5418^{\circ} \mathrm{A}\right) \mathrm{X}$-ray source, operating at $35.4 \mathrm{kV}$ and $28 \mathrm{~mA}$. Textural properties of the synthesized samples were measured by nitrogen adsorption at $77 \mathrm{~K}$ using a BELSORP-max apparatus after being out-gassed in vacuum at 120 ${ }^{\circ} \mathrm{C}$. Elemental analysis was carried out using CHNSElementar. FT-IR spectra was recorded using a FTIR 70 VERTX Bruker spectrophotometer using $\mathrm{KBr}$ powder. UV-Vis spectrophotometer was used to measure the concentration of the Methylene Blue and Direct red23 in aqueous solution. FESEM images were captured using Mira TESCAN 3-XMU. Samples were sputtered with gold prior to imaging. TEM images were recorded at $150 \mathrm{kV}$ operating voltage using a Zeiss microscope.

\section{Results and discussion}

\subsection{Characterization of $\mathrm{NH}_{2} / \mathrm{UVM}-7 / \mathrm{SO}_{3} \mathrm{H}$} $\mathrm{XRD}$ pattern of $\mathrm{NH}_{2} / \mathrm{UVM}-7 / \mathrm{SO}_{3} \mathrm{H}$ is shown in Figure 2. 
A strong and broad diffraction peak can be observed which is characterized in bimodal meso/macroporous material. This peak can be attributed to diffraction of incident $\mathrm{X}$ ray from the $d_{100}$ plane. According to the hexagonal symmetry, two weak and broad peaks at higher diffraction angles can be assigned to $d_{110}$ and overlap of $d_{200}$ and $d_{210}$ planes, respectively.

\subsection{Nitrogen physisorption}

The isotherms of UVM-7 and $\mathrm{NH}_{2} / \mathrm{UVM}-7 / \mathrm{SO}_{3} \mathrm{H}$ is shown in Figure 3. The isotherms showed the four type of isotherm according to the IUPAC classification. In both isotherms, at low relative pressure, the inflection is not sharp. By approaching to the saturated relative pressure, increasing of gas uptake led to the sharp increasing of isotherm. This can be attributed to the inter particles of UVM type material which is also present in $\mathrm{NH}_{2} / \mathrm{UVM}-7 /$ $\mathrm{SO}_{3} \mathrm{H}$ after internal and external grafting of organic moieties. The high BET surface area and pore volume means that the pore structure/size almost preserved after grafting. However, after grafting of organic moieties on UVM-7, the sharpness of these region slightly decreases. The two inflections in $\mathrm{NH}_{2} / \mathrm{UVM}-7 / \mathrm{SO}_{3} \mathrm{H}$ can be attributed to the presence of two types of pore system.

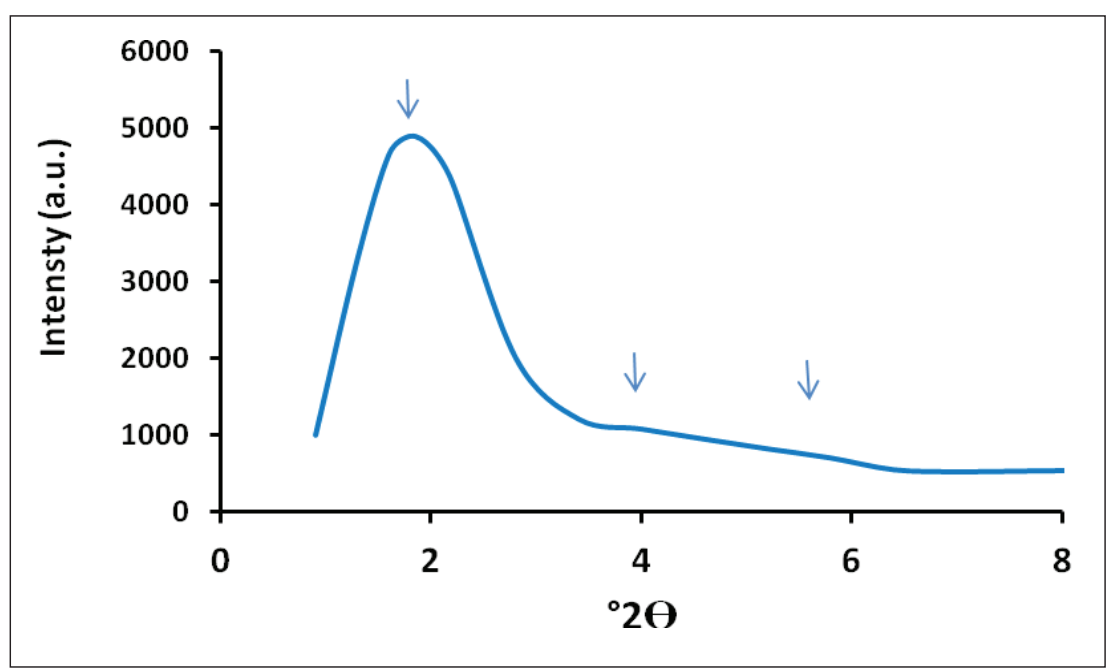

Fig. 2. The XRD pattern of $\mathrm{NH}_{2} / \mathrm{UVM}-7 / \mathrm{SO}_{3} \mathrm{H}$

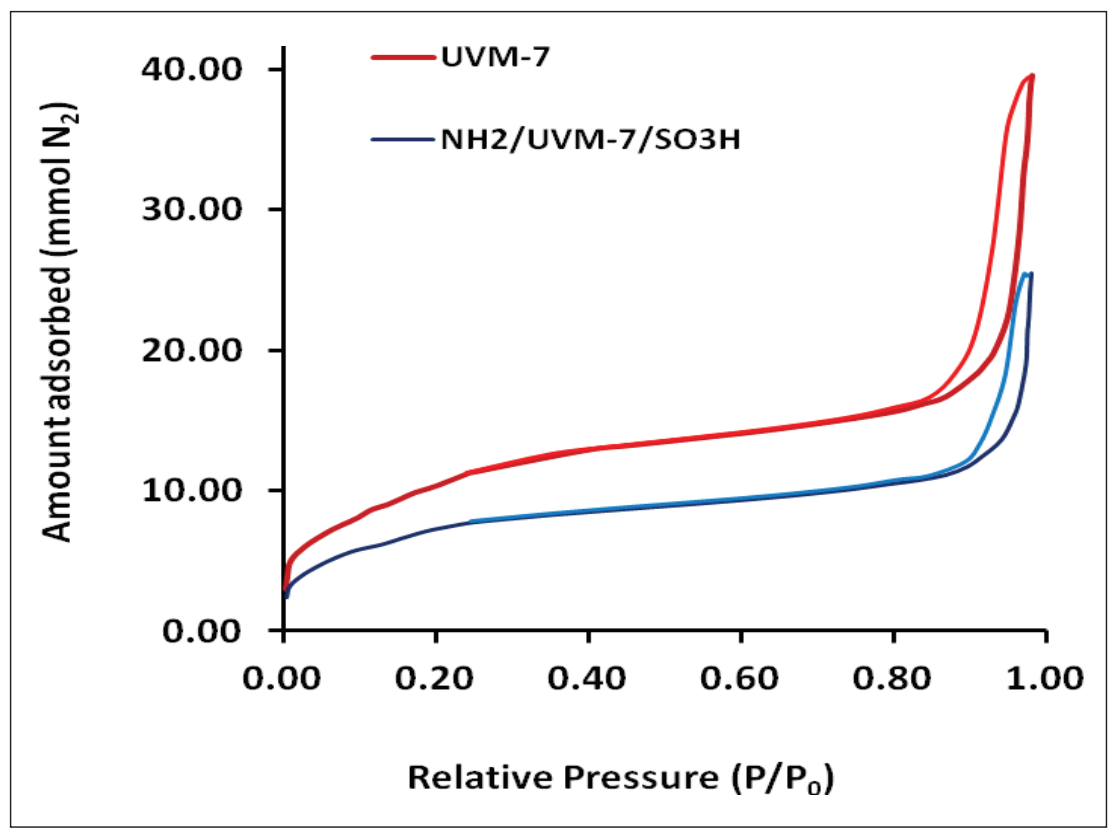

Fig. 3. The isotherms of UVM-7 and $\mathrm{NH}_{2} / \mathrm{UVM}-7 / \mathrm{SO}_{3} \mathrm{H}$ 


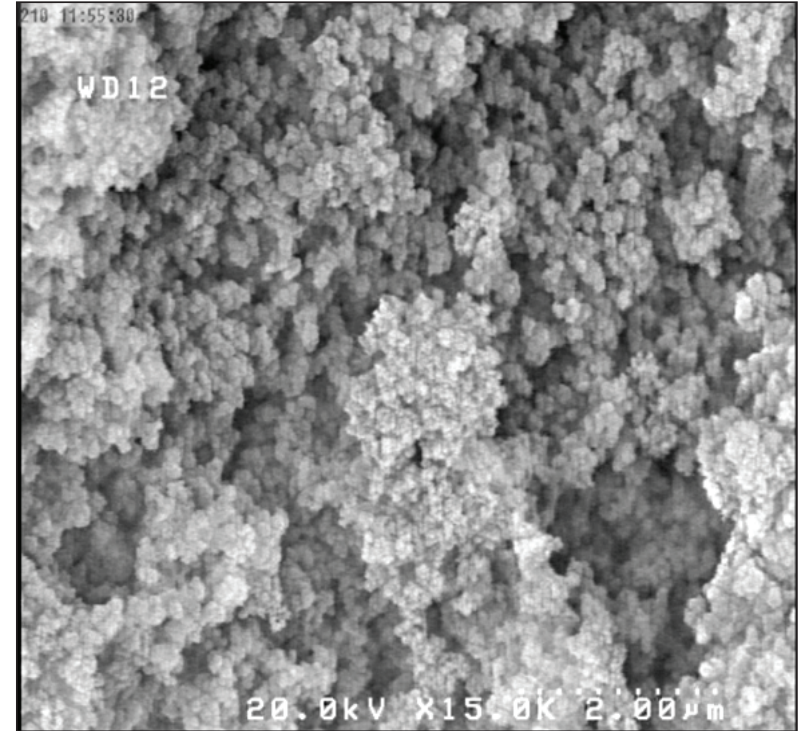

Fig.4. The $\mathrm{SEM}$ of $\mathrm{NH}_{2} / \mathrm{UVM}-7 / \mathrm{SO}_{3} \mathrm{H}$

\subsection{TEM and SEM}

Electron microscopy is a powerful technique provides a direct view of porous structure, particle morphology and many other information of porous materials at nanoscale. The SEM of $\mathrm{NH}_{2} /$ UVM-7/ $\mathrm{SO}_{3} \mathrm{H}$ reveals the particle size, shape and morphology of nanomaterials. Figure 4 illustrates the SEM image of $\mathrm{NH}_{2} / \mathrm{UVM}-7 / \mathrm{SO}_{3} \mathrm{H}$. The sample composed of nanosize particles without presence of large agglomerates. The shape of the nanoparticles is almost irregular. The TEM image of the $\mathrm{NH}_{2} / \mathrm{UVM}$ $7 / \mathrm{SO}_{3} \mathrm{H}$ is taken perpendicular to $d_{100}$ direction of pores and is displayed in Figure 5. Pore openings are clearly visible. Particle size in sub-ten nanometers and is in agreement with those seen in SEM image.

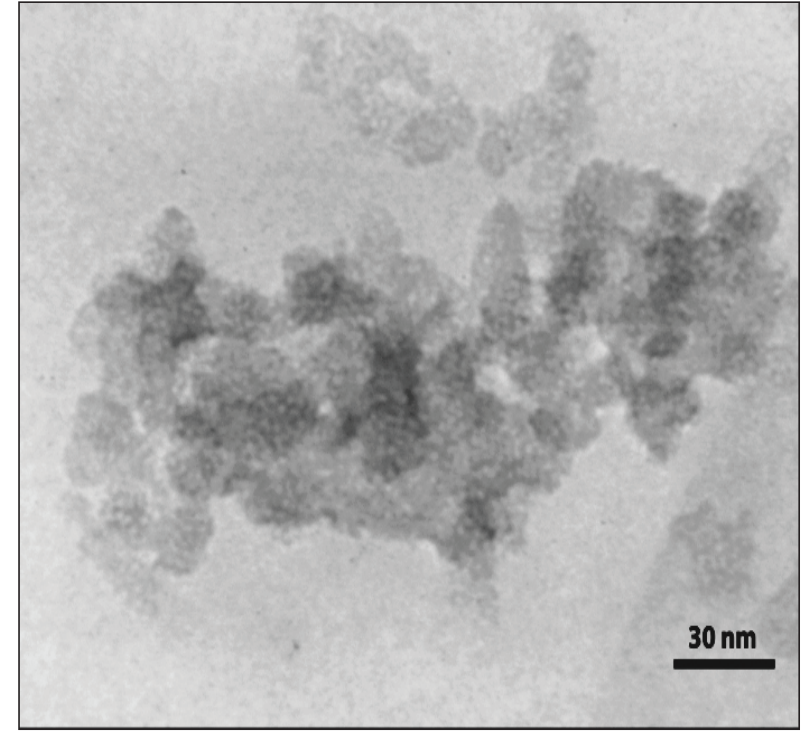

Fig.5. The TEM of $\mathrm{NH}_{2} / \mathrm{UVM}-7 / \mathrm{SO}_{3} \mathrm{H}$

FT-IR spectra of $\mathrm{SO}_{3} \mathrm{H} / \mathrm{UVM}-7 / \mathrm{NH}_{2}$ is shown in Figure 6. FT-IR spectroscopy was utilized to indicate the existence of $\mathrm{NH}_{2}$ and $\mathrm{SO}_{3} \mathrm{H}$ functional groups on the UVM-7. Two distinct peaks around $457 \mathrm{~cm}^{-1}$ and $1082 \mathrm{~cm}^{-1}$ is attributed to the symmetric and asymmetric stretching bands of Si-O-Si group in the framework of UVM-7. A broad peak at around 3451 $\mathrm{cm}^{-1}$ is assign to the silanol groups on the surface of UVM-7. A small absorption band at $1558 \mathrm{~cm}^{-1}$ is attributed to the bending vibration of amine group which overlapped with symmetric bending vibrations of O-H. A stretching mode of $\mathrm{NH}_{2}$ is also overlapped with silanol group's around $3451 \mathrm{~cm}^{-1}$. This indicates that the UVM-7 possess of $\mathrm{NH}_{2}$ groups. A shoulder, overlapped with asymmetric stretching of Si-O-Si,

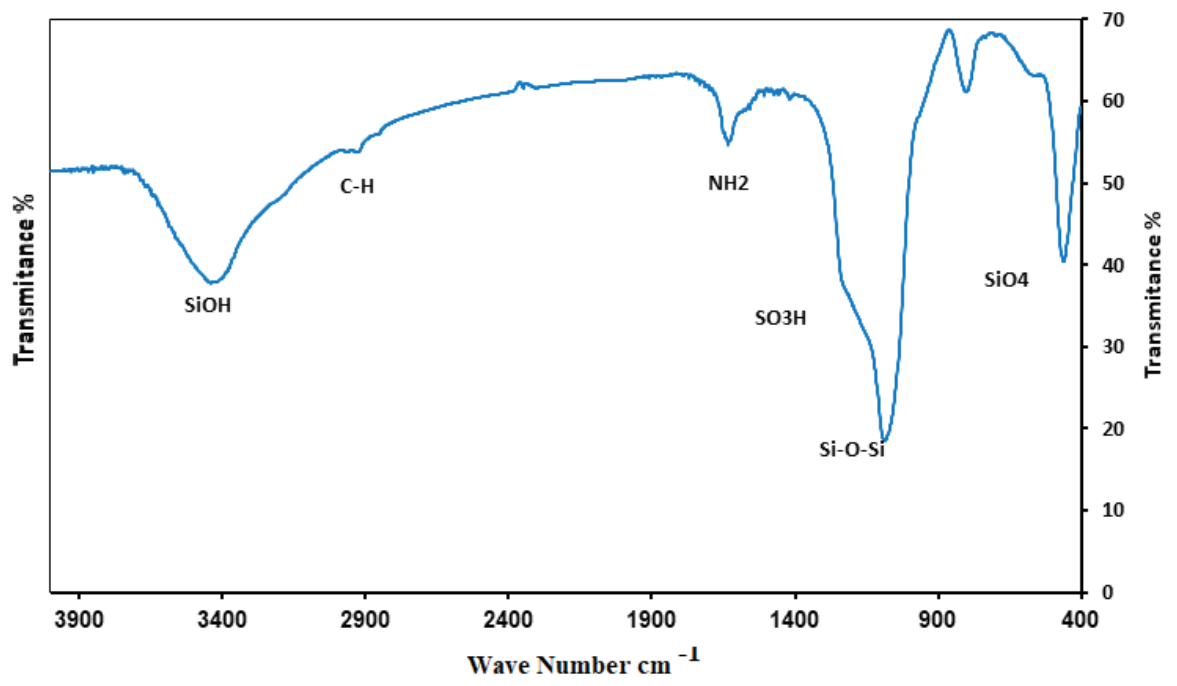

Fig. 6. The FT-IR spectra of $\mathrm{SO}_{3} \mathrm{H} / \mathrm{UVM}-7 / \mathrm{NH}_{2}$ 
is attributed to the $\mathrm{SO}^{3-}$ and $\mathrm{S}=\mathrm{O}$ stretching mode of sulfonic acid functional group. It cannot be seen absorption mode of S-H group in the entire spectrum means that thiol groups were fully oxidized to sulfonic acid. Two small absorption peaks at 2852 $\mathrm{cm}^{-1}$ and $2926 \mathrm{~cm}^{-1}$ is characteristic of the stretching vibration mode of methylene groups of the propyl chain of the APTES and TPTES.

These results approve coexistence of amine and sulfonic acid groups on UVM-7 simultaneously. This statement is supported by elemental analysis of $\mathrm{NH}_{2} / \mathrm{UVM}-7 / \mathrm{SO}_{3} \mathrm{H}$. Elemental analysis of $\mathrm{NH}_{2} /$ $\mathrm{UVM}-7 / \mathrm{SO}_{3} \mathrm{H}$ revealed that the sample contains $4.9 \%(\mathrm{w} / \mathrm{w}$ basis) of nitrogen and $5.0 \%$ of sulfur.
These results proof presence of amine and sulfonic acid functional groups on $\mathrm{NH}_{2} / \mathrm{UVM}-7 / \mathrm{SO}_{3} \mathrm{H}$.

\subsection{Determination of MB and Dr in single and binary solution}

The calibration curve obtained from absorbance spectra of MB and Dr versus concentration at $\lambda_{\text {max }}$ of the corresponding dye and used for the determination of dyes in single solution. The concentration of dyes in binary solution of MB and Dr is obtained by first order derivative spectrophotometry by plotting the change rate of absorbance against wavelength. The obtained spectra for raw and derivative spectra are displayed in Figure 7a and 7b.

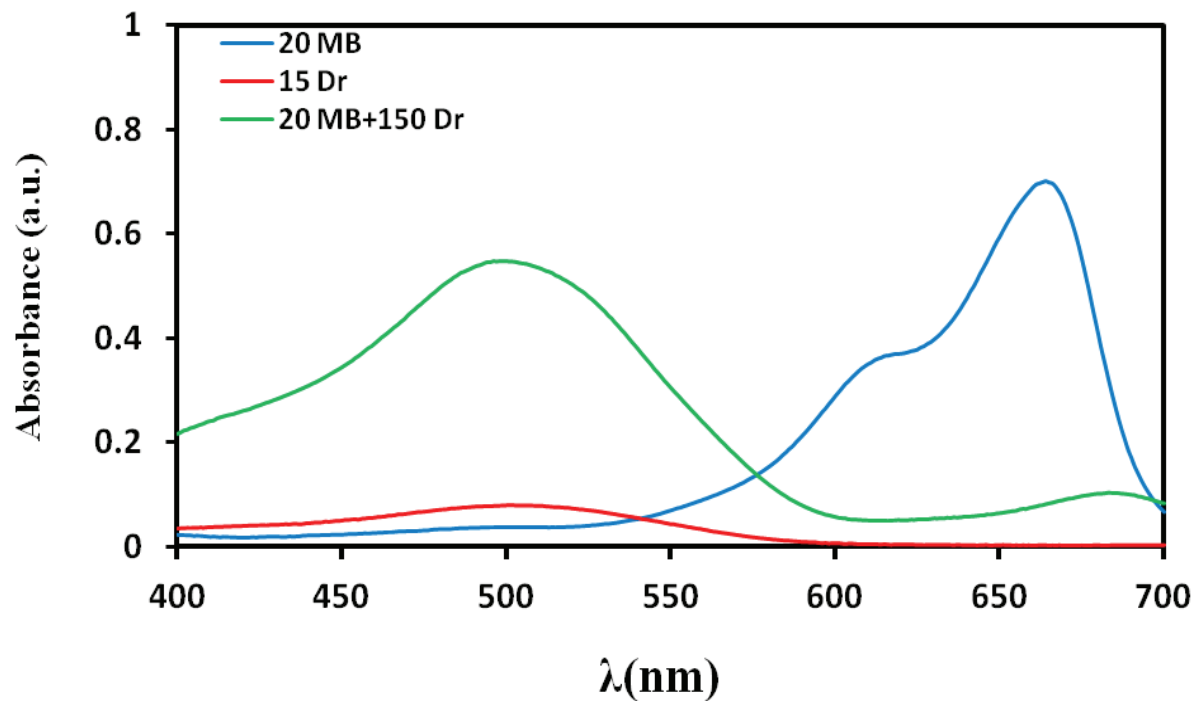

Fig. 7a. The Raw absorption spectra of: $\mathrm{MB}\left(20 \mathrm{mg} \mathrm{L}^{-1}\right)$, Dr $\left(15 \mathrm{mg} \mathrm{L}^{-1}\right)$, and their mixture at $\lambda_{\max }$ of the corresponding dye

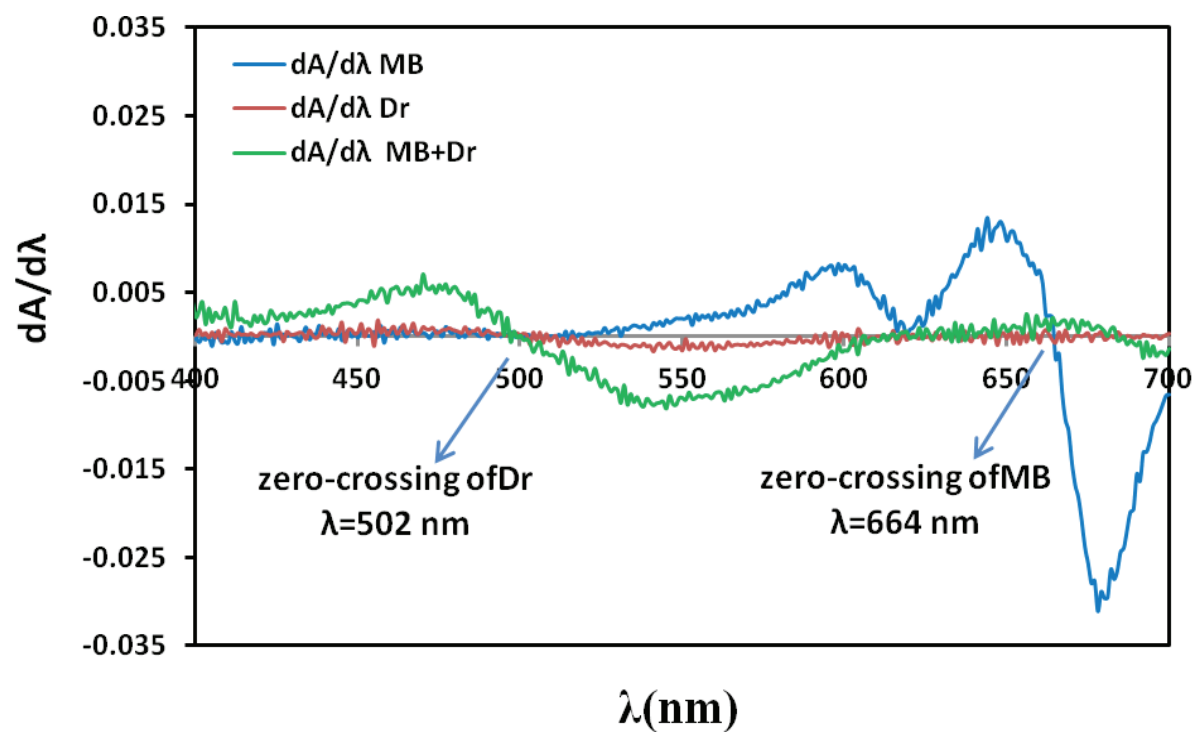

Fig. 7b. The first derivative spectra of MB, Dr, and their mixture by plotting the change rate of absorbance against wavelength 
The calibration curves for determination of each dye in the presence of the other one were obtained by measuring of first-order derivative at the zero crossing points of $\operatorname{Dr}(502 \mathrm{~nm})$ and MB $(664 \mathrm{~nm})$ $[27,28]$. Several standard binary mixtures of MB and Dr were used for the validation of the method. The regression equations and coefficients of determinations, are given in Table 1.

\subsection{Equilibrium modeling}

\subsubsection{Single-component adsorption isotherm}

Two well-known adsorption model, Langmuir and Freundlich, were applied to the adsorption data of single-component dye solution (Equation 3 and 4) $[29,30]$. In single solution, the $R^{2}$ of Langmuir isotherm was higher than that of Freundlich, which implies that suggestions of Langmuir model. The homogenous adsorption sites, equal activation energy and monolayer formation, is predominantly determine the mechanism of adsorption. The isotherm constants are presented in Table 2.

$$
\frac{\mathcal{C}_{e}}{q_{e}}=\frac{1}{b q_{m}}+\frac{\mathcal{C}_{e}}{q_{m}}
$$

$$
\log q_{e}=\log K_{f}+\frac{1}{n} \log q_{m} \quad \text { (Eq. 4) }
$$

\subsubsection{Multi-component adsorption isotherms}

In this case, competition for occupation of adsorption sites by adsorbate molecules occur and

Table 1. Determination of MB and Dr in mixture solution using zero-derivative and first-derivative spectrophotometry

\begin{tabular}{ccccc}
\hline Solution No. & $* \mathbf{M B}\left(\mathbf{m g ~ L}^{-1}\right)$ & $* \mathbf{D r}\left(\mathbf{m g ~ L}^{-1}\right)$ & ${ }^{a}$ Regression equations & $\mathbf{R}^{2}$ \\
\hline 1 & $1-5$ & 0 & $\mathrm{~S}=0.008 \mathrm{C}+0.1841$ & 0.9981 \\
2 & $2-20$ & 10 & $\mathrm{~B}=0.0015 \mathrm{C}+0.002$ & 0.9890 \\
3 & 0 & $1-10$ & $\mathrm{~S}=0.0471 \mathrm{C}+0.021$ & 0.9974 \\
4 & 2 & $10-25$ & $\mathrm{~B}=0.0001 \mathrm{C}-0.0006$ & 0.9963 \\
\hline
\end{tabular}

*C is the concentration of the corresponding dye $\left(m g L^{-1}\right)$

\begin{tabular}{|c|c|c|c|c|}
\hline Langmuir & $q e(m g g-1)$ & $b(m g ~ L-1)$ & \multicolumn{2}{|c|}{$\mathbf{R 2}$} \\
\hline $\mathrm{MB}(\sin )$ & 52.63 & 0.226 & \multicolumn{2}{|c|}{0.987} \\
\hline $\operatorname{Dr}(\sin )$ & 88.33 & 0.0983 & \multicolumn{2}{|c|}{0.996} \\
\hline MB(bin) & 18.86 & 0.0096 & \multicolumn{2}{|c|}{0.876} \\
\hline Dr(bin) & 100 & 0.1315 & \multicolumn{2}{|c|}{0.977} \\
\hline Frendlich & Kf(mg g-1) & $\mathbf{n}$ & & \\
\hline $\mathrm{MB}(\sin )$ & 0.0272 & 3.412 & \multicolumn{2}{|c|}{0.947} \\
\hline $\operatorname{Dr}(\sin )$ & 23.28 & 3.984 & \multicolumn{2}{|c|}{0.950} \\
\hline MB(bin) & 98.24 & 2.32 & \multicolumn{2}{|c|}{0.958} \\
\hline $\operatorname{Dr}($ bin $)$ & 172.60 & 5.29 & \multicolumn{2}{|c|}{0.806} \\
\hline Extended-Langmuir & $q \max (\operatorname{mg} g-1)$ & b1 & b2 & $\mathbf{R 2}$ \\
\hline MB(bin) & 74.32 & 0.0021 & 0.0038 & 0.999 \\
\hline $\operatorname{Dr}($ bin $)$ & 106.78 & 0.0043 & 0.0056 & 0.998 \\
\hline
\end{tabular}

${ }^{a} S$ and $B$ is the response in single and binary solution

Table 2. Parameters of Langmuir and Freundlich models in single and binary solutions.

Condition: $10 \mathrm{~mL}$ solution, $\mathrm{pH}: 4$ in single and binary solutions. 
led to a complex adsorption model. Furthermore, for the design of treatment/refining systems, understanding the mechanism of multi-component adsorption is very important. Despite the difficulty of obtaining of a model for adsorption of binary mixtures, extended-Langmuir model was developed to explain equilibrium adsorption model in such system is as Equation 5:

$q_{e, i}=\frac{q_{i} b_{i} c_{i}}{1+\sum b_{j} c_{j}} \times 100 \quad$ (Eq. 5)

Using non-linear regression technique, parameters of Equation 5 were obtained given in Table 2. High regression coefficients ( $\left.\mathrm{R}^{2}: 96.98-99.86\right)$ indicate that the extended Langmuir model has the ability to explain the adsorption equilibrium of binary solution of MB and Dr.

\subsection{Adsorption kinetic}

The kinetic data of adsorption was obtained with the initial concentrations of $50 \mathrm{mg} \mathrm{L}^{-1}$ and $100 \mathrm{mg}$ $\mathrm{L}^{-1}$ for $\mathrm{MB}$ and $\mathrm{Dr}$, respectively. Solutions prepare in $25 \mathrm{~mL}$ flask with the stirring rate of $300 \mathrm{rpm}$ and the contact time was changed in the range of 10 to 120 minutes. to evaluate if there are changes/ interferences in the adsorption process in the presence of other dyes in the solution.

\subsubsection{Pseudo-first order model}

The pseudo- first order kinetic model has been widely used to explain kinetic of adsorption. The model was described by the following Equation 6:

$$
\frac{1}{q_{t}}=\frac{k_{1}}{q_{e}}\left(\frac{1}{t}\right)+\frac{1}{q_{e}}
$$

Where $\mathrm{q}_{\mathrm{e}}$ and $\mathrm{q}_{\mathrm{t}}$ are the adsorption capacities $\left(\mathrm{mg} \mathrm{g}^{-1}\right)$ at equilibrium and at time $t$, respectively. $K_{1}$ is the rate constant of pseudo-first order adsorption $\left(\mathrm{L} \mathrm{min}^{-1}\right)$. The pseudo-second order rate equation of McKay and Ho can be represented in the Equation 7.

$$
\frac{t}{q}=\frac{1}{k_{2} q^{2}}+\frac{1}{q} t
$$

Where $\mathrm{q}_{\mathrm{e}}$ is the equilibrium adsorption capacity, and $\mathrm{K}_{2}$ is the pseudo-second order constants $\left(\mathrm{g} \mathrm{mg}^{-1} \cdot \mathrm{min}^{-1}\right)$. These terms can be determined experimentally from the slope and intercept of plot $t / q_{t}$ versus $t$, shown in Figure 8. The coefficients of both models are shown in Table 3. In all cases including single and binary solutions, the pseudosecond order model has better coefficient of determination, $\mathrm{R}^{2}$, which means that kinetic of adsorption od $\mathrm{MB}$ and $\mathrm{Dr}$ on $\mathrm{NH}_{2} / \mathrm{UVM}-7 / \mathrm{SO}_{3} \mathrm{H}$ can be explained by The difference between two kinetic model can be attributed to the number and accessibility of adsorption sites, specific surface area, pore size, nature of the functional groups of adsorbent and chemical structure of dyes.

Table 3. Kinetics parameters values for the adsorption of $\mathrm{MB}$ and $\mathrm{Dr}$ on $\mathrm{NH}_{2}-\mathrm{SO}_{3} \mathrm{H} / \mathrm{UVM}-7$ in single ( $\sin$ ) and binary (bin) dyes solution

\begin{tabular}{ccccccc}
\hline & \multicolumn{3}{c|}{ Pseudo-first order } & \multicolumn{3}{c}{ Pseudo-second order } \\
\cline { 2 - 7 } & $\mathrm{q}_{\mathrm{e}}\left(\mathrm{mg} \mathrm{g}^{-1}\right)$ & $\mathrm{K}_{1}(\mathrm{~min})$ & $\mathrm{R}^{2}$ & $\mathrm{q}_{\mathrm{e}}\left(\mathrm{mg} \mathrm{g}^{-1}\right)$ & $\mathrm{K}_{2}\left(\mathrm{~g} \mathrm{mg}^{-1} \cdot \mathrm{min}^{-1}\right)$ & $\mathrm{R}^{2}$ \\
\hline $\mathrm{MB}(\sin )$ & 40 & 3.7 & 0.867 & 50 & 0.0012 & 0.962 \\
$\operatorname{Dr}(\sin )$ & 4.3 & 2.43 & 0.765 & 5.26 & 0.372 & 0.983 \\
$\mathrm{MB}(\mathrm{bin})$ & 15.87 & 2.142 & 0.876 & 12.98 & 0.082 & 0.934 \\
$\operatorname{Dr}($ bin $)$ & 66.66 & 1 & 0.765 & 90.9 & 0.00068 & 0.826 \\
\hline
\end{tabular}



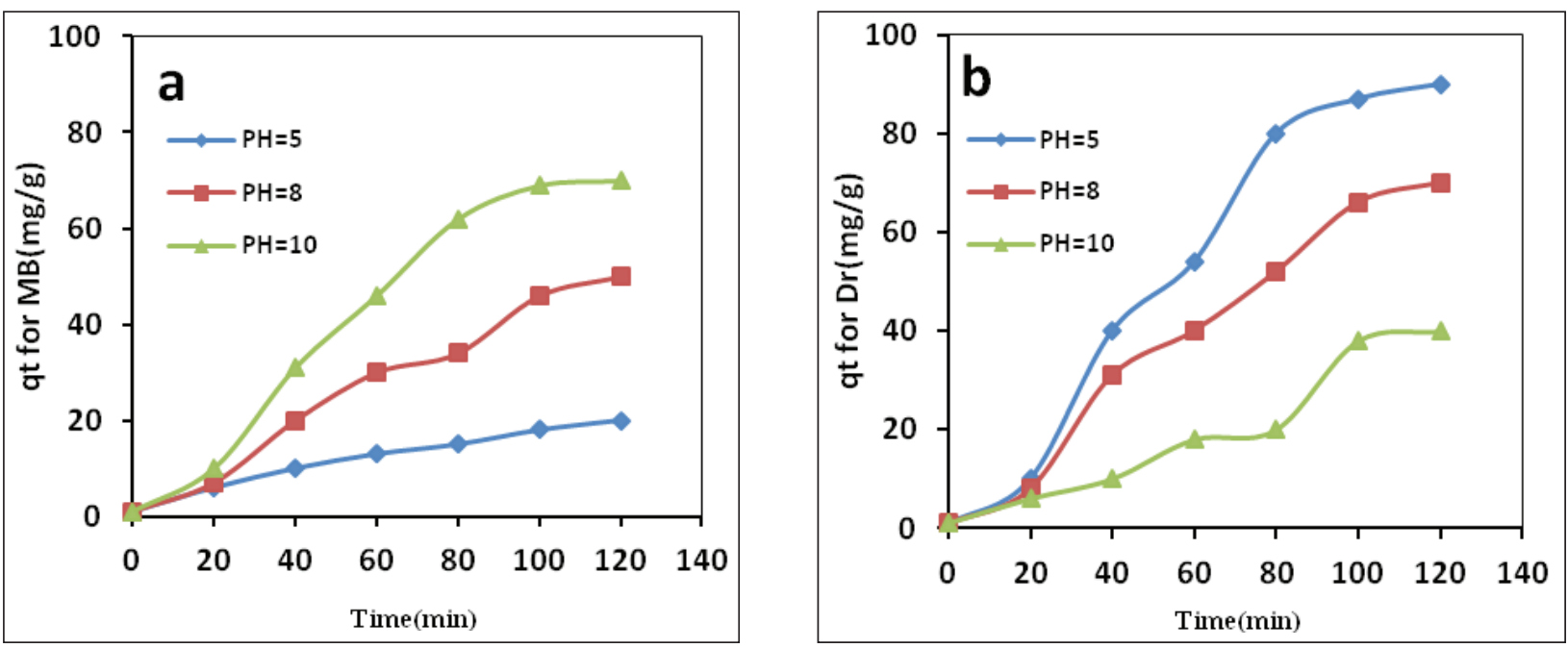

Fig. 8. The effect of pretreatment $\mathrm{pH}$ on selective adsorption of MB (a)

and $\mathrm{Dr}$ (b) on $\mathrm{NH}_{2}-\mathrm{SO}_{3} \mathrm{H} / \mathrm{UVM}-7$ in binary solution (temperature $30^{\circ} \mathrm{C}, 400 \mathrm{mgL}^{-1}$ initial dye concentration).

\subsubsection{Selective adsorption of cationic and anionic dyes}

Figure 8 displays that $\mathrm{NH}_{2} / \mathrm{UVM}-7 / \mathrm{SO}_{3} \mathrm{H}$ can selectively adsorb the anionic Dr from the $\mathrm{Dr} / \mathrm{MB}$ mixture and pretreated at $\mathrm{pH}=5$. While, the $\mathrm{NH}_{2} /$ $\mathrm{UVM}-7 / \mathrm{SO}_{3} \mathrm{H}$ can selectively adsorb the cationic MB dye from the Dr/MB mixture and pretreated at $\mathrm{pH}=10$. This test verifies with the easy, the special treatment and selective adsorption of cationic or anionic dyes by $\mathrm{NH}_{2}-\mathrm{SO}_{3} \mathrm{H} / \mathrm{UVM}-7$.

\subsubsection{Thermodynamic studies}

The effect of temperature on the adsorption of $\mathrm{MB}$ and $\mathrm{Dr}$ onto $\mathrm{NH}_{2} / \mathrm{UVM}-7 / \mathrm{SO}_{3} \mathrm{H}$ was investigated. The obtained results indicate that the amount of equilibrium adsorption (qe) of $\mathrm{MB}$ and $\mathrm{Dr}$ on the $\mathrm{NH}_{2} / \mathrm{UVM}-7 / \mathrm{SO}_{3} \mathrm{H}$ slightly increases with increasing temperature which reveals the endothermic nature of adsorption. Main thermodynamic parameters including standard free energy $(\Delta \mathrm{G})$, enthalpy $(\Delta \mathrm{H})$ and entropy $(\Delta \mathrm{S})$ were obtained using Equation 8.

$$
\ln k c=\frac{-\Delta H}{R T}+\frac{\Delta S}{R} \quad \text { (Eq. 8) }
$$

Where $\mathrm{R}$ is gas constant $\left(8.314 \mathrm{Jmol}^{-1} \mathrm{~K}^{-1}\right)$ and $\mathrm{T}$ is temperature in Kelvin. The apparent equilibrium constant $\left(\mathrm{K}_{c}\right)$ of the adsorption is defined as Equation 9.

$$
k_{c}=\frac{\mathcal{C}_{a}}{\mathcal{C}_{b}}
$$

Where $\mathrm{C}_{\mathrm{a}}$ and $\mathrm{C}_{\mathrm{b}}$ is the equilibrium concentration (mg L $\mathrm{L}^{-1}$ ) a of corresponding dye on the adsorbent and in the solution, respectively. The $\mathrm{K}_{\mathrm{c}}$ value is used in the Equation 10 to determine the Gibbs free energy of adsorption $(\Delta \mathrm{G})$.

$$
\Delta G=-R T \ln K c
$$

Furthermore, $\Delta \mathrm{H}$ and $\Delta \mathrm{S}$ were calculated from the slope and intercept of the linear plot of $\operatorname{lnKc}$ versus $1 / T$, respectively. The obtained thermodynamic parameters are summarized in Table 4 . All $\Delta \mathrm{G}$ values are negative which imply that the spontaneous nature of adsorption. Furthermore, the value of $\Delta \mathrm{G}$ becomes more negative with increasing of temperature, indicating that the adsorption process is more favorable at higher temperature. As can be seen in Table 4, the positive value of adsorption enthalpy, $\Delta \mathrm{S}$, confirms the endothermic nature the adsorption process. 
Table 4. Values of thermodynamic parameters for dye adsorption from binary solution onto $\mathrm{NH}_{2} / \mathrm{UVM}-7 / \mathrm{SO}_{3} \mathrm{H}$

\begin{tabular}{|c|c|c|c|c|}
\hline Dye & Temp.(K) & $\Delta G\left(\mathrm{~kJ} \mathrm{~mol}^{-1}\right)$ & $\Delta \mathbf{H}\left(\mathrm{kJ} \mathrm{mol}^{-1}\right)$ & $\Delta \mathrm{S}\left(\mathrm{kJ} \mathrm{mol}^{-1} \mathbf{k}^{-1}\right)$ \\
\hline \multirow{5}{*}{ MB } & 298 & -2.95 & 8.1 & 0.038 \\
\hline & 308 & -3.21 & & \\
\hline & & & & \\
\hline & 318 & -3.43 & & \\
\hline & 328 & -3.98 & & \\
\hline \multirow{4}{*}{ Dr } & 298 & -4.83 & 26.3 & 0.106 \\
\hline & 308 & -5.82 & & \\
\hline & 318 & -6.98 & & \\
\hline & 328 & -7.83 & & \\
\hline
\end{tabular}

\section{Conclusions}

In This work, a novel synthesis method was developed for the preparation of an adsorbent contains both sulfonic acid and basic amine groups in the exterior and interior pores of UVM-7, respectively. This multifunctional adsorbent, $\mathrm{NH}_{2} / \mathrm{UVM}-7 / \mathrm{SO}_{3} \mathrm{H}$, can be used for simultaneous removal of multiple dyes with different electrostatic charge. In real aqueous sample and wastewater there are several dyes which differ in their nature. Furthermore, one of the most effective parameters in the removal of dye is its electrostatic charge. On the other hand, large pore size and surface area of UVM-7 can improve the diffusion of the dye toward the adsorption sites. This improved diffusion rate can be accompanied by small size of the adsorbent particles, UVM-7, to improve adsorption yield. However, it would be very useful in terms of industrial point of view, to have a single adsorbent to remove a variety of pollutants. The proposed method can be applied to use a variety of adsorbents and open us a way for treatment of wastewaters which normally contain many types of pollutants.

\section{Acknowledgments}

The authors would like to thank from Faculty of chemistry, Amirkabir University of Technology, Tehran, Iran.

\section{References}

[1] F. I. Vacchi, A. F. Albuquerque, J. A. Vendemiatti, D. A. Morales, A. B. Ormond, H. S. Freeman, G. J. Zocolo, M. V. B. Zanoni, G. Umbuzeiro, Chlorine disinfection of dye wastewater: Implications for a commercial azo dye mixture, Sci. Total Environ., 442 (2013) 302-309.

[2] M. Anbia, S. A. Hariri, Removal of methylene blue from aqueous solution using nanoporous SBA-3, Desalination, 261 (2010) 61-66.

[3] M. T. Yagub, T. K. Sen, S. Afroze, H. M. Ang, Dye and its removal from aqueous solution by adsorption: a review, Adv. Colloid Interface Sci., 209 (2014) 172-184.

[4] H. Chaudhuri, S. Dash, A. Sarkar, SBA-15 functionalised with high loading of amino or carboxylate groups as selective adsorbent for enhanced removal of toxic dyes from aqueous solution, New J. Chem., 40 (2016) 3622-3634.

[5] Z. Wu, Q. Lu, W. H. Fu, S. Wang, C. Liu, N. Xu, D. Wang, Y. M. Wang, Z. Chen, Fabrication of mesoporous Al-SBA-15 as a methylene blue capturer via a spontaneous infiltration route, New J. Chem., 39 (2015) 985-993. 
[6] B. Coasne, Multiscale adsorption and transport in hierarchical porous materials, New J. Chem., 40 (2016) 4078-4094.

[7]J. F. Gao, J. H. Wang, C. Yang, S. Y. Wang, Y. Z. Peng, Binary biosorption of Acid Red 14 and Reactive Red 15 onto acid treated okara: Simultaneous spectrophotometric determination of two dyes using partial least squares regression, Chem. Eng. J., 71 (2011) 967-975.

[8] J. F. Gao, Q. Zhang, K. Su, J. H. Wang, Competitive biosorption of Yellow 2G and Reactive Brilliant Red K-2G onto inactive aerobic granules: simultaneous determination of two dyes by first-order derivative spectrophotometry and isotherm studies, Bioresour. Technol., 101 (2010) 5793-5801.

[9] V. K. Gupta, B. Gupta, A. Rastogi, S. Agarwal, A. Nayak, A comparative investigation on adsorption performances of mesoporous activated carbon prepared from waste rubber tire and activated carbon for a hazardous azo dye--Acid Blue 113, J. Hazard. Mater., 186 (2011) 891-901.

[10] P. K. Malik, S. K. Saha, Oxidation of direct dyes with hydrogen peroxide using ferrous ion as catalyst, Sep. Purif. Technol., 31 (2003) 241-250.

[11] M. Anbia, S. A. Hariri, S. N. Ashrafizadeh, Adsorptive removal of anionic dyes by modified nanoporous silica SBA3, Appl. Surf. Sci., 256 (2010) 3228-3233.

[12] S. Wang, H. Li, L. Xu, Application of zeolite MCM-22 for basic dye removal from wastewater, J. Colloid Interface Sci., 295 (2006) 71-78.

[13] G. Ciardelli, L. Corsi, M. Marcucci, Membrane Separation for Wastewater Reuse in the Textile Industry, Resour. Conserv. Recycl., 31 (2001) 189-197

[14] N. Suzuki, J. Liu, Y. Yamauchi, Recent progress on the tailored synthesis of various mesoporous fibers toward practical applications, New J. Chem., 38 (2014) 3330-3335.
[15] M. Turabik, Adsorption of basic dyes from single and binary component systems onto bentonite: Simultaneous analysis of Basic Red 46 and Basic Yellow 28 by first order derivative spectrophotometric analysis method, J. Hazard. Mater., 158 (2008) 5264.

[16] M. Kucharska, J. Grabka, A review of chromatographic methods for determination of synthetic food dyes, Talanta, 80 (2010) 1045-1051.

[17] A. A. Peláez-Cid, S. Blasco-Sancho, F. M. Matysik, Determination of textile dyes by means of non-aqueous capillary electrophoresis with electrochemical detection, Talanta, 75 (2008) 1362-1368.

[18]C. Bosch Ojeda, F. Sanchez Rojas, Recent applications in derivative ultraviolet/visible absorption spectrophotometry: 2009-2011: A review, Microchem. J., 106 (2013) 1-16.

[19] F. Sánchez Rojas, C. Bosch Ojeda, Recent development in derivative ultraviolet/visible absorption spectrophotometry: 2004-2008: a review, Anal. Chim. Acta, 635 (2009) 22-44.

[20] C. Bosch Ojeda, F. Sanchez Rojas, Recent developments in derivative ultraviolet/ visible absorption spectrophotometry, Anal. Chim. Acta, 518 (2004) 1-24.

[21] J. Zolgharnein, M. Bagtash, T. Shariatmanesh, Simultaneous removal of binary mixture of Brilliant Green and Crystal Violet using derivative spectrophotometric determination, multivariate optimization and adsorption characterization of dyes on surfactant modified nano- $\gamma$-alumina, Spectrochim. Acta - Part A Mol. Biomol. Spectrosc., 137 (2015) 1016-1028.

[22] A. Vargas and M. Reyes, Integral solutions to complex problems: climate change, adaptation policies and payment for ecosystem services schemes, Int. J. Plur. Econ. Educ., 3 (2012) 173.

[23] H. Shirkhanloo, A. Khaligh, F. Golbabaei, Z. Sadeghi, A .Vahid, A. Rashidi, On-line 
micro column preconcentration system based on amino bimodal mesoporous silica nanoparticles as a novel adsorbent for removal and speciation of chromium (III, VI) in environmental samples, J. Environ. Health Sci. Eng. 13 (2015) 1-12.

[24] H. Shirkhanloo, S.D. Ahranjani, A lead analysis based on amine functionalized bimodal mesoporous silica nanoparticles in human biological samples by ultrasound assisted-ionic liquid trap-micro solid phase extraction, Journal of pharm. Biomed. Anal.,157 (2018) 1-9.

[25] H. Shirkhanloo, M. Falahnejad, H.Z. Mousavi, On-line ultrasound-assisted dispersive micro-solid-phase extraction based on amino bimodal mesoporous silica nanoparticles for the preconcentration and determination of cadmium in human biological samples, Biol. Trace Elem. Res., 171 (2016) 472-481.

[26] H. Shirkhanloo, M. Falahnejad, H.Z. Mousavi, Mesoporous silica nanoparticles as an adsorbent for preconcentration and determination of trace amount of nickel in environmental samples by atom trap flame atomic absorption spectrometry, J. Appl. Spec., 82 (2016) 1072-1077.

[27] C.P. Ye, R. N. Wang, X. Gao, W.Y. Li, $\mathrm{CO}_{2}$ Capture Performance of supported phosphonium dual amine-functionalized ionic liquids@MCM-41, Energy Fuels, 34 (2020) 14379-14387.

[28] A. Balcha, P.O. Yadav, T. Dey, Photocatalytic degradation of methylene blue dye by zinc oxide nanoparticles obtained from precipitation and sol-gel methods, Environ. Sci. Pollut. Res. Int., 23 (2016) 25485-25493.

[29] I. Langmuir, THE adsorption of gases on plane surfaces of glass, mica and platinum, J. Am. Chem. Soc., 40 (1918) 1361-1403.

[30] J.Yu,A.Zou,W.He,B.Liu,Adsorptionofmixed dye system with cetyltrimethylammonium bromide modified sepiolite: characterization, performance, kinetics and thermodynamics, Water, 12 (2020) 981. doi:10.3390/w12040981 